\title{
Análisis de los significados del concepto de diferencial desde una perspectiva ontosemiótica
}

\author{
Manuel Alejandro Verón \\ Belén Giacomone
}

\begin{abstract}
Resumen: En esta investigación se aborda el estudio de los diversos significados del concepto de diferencial aplicando herramientas teóricas del Enfoque Ontosemiótico, en particular las nociones de significado pragmático de un concepto y configuración de prácticas, objetos y procesos. El objetivo es construir un modelo ontosemiótico de referencia; para ello, en primer lugar, se realiza un estudio histórico-epistemológico sobre el origen y evolución del diferencial, identificando cuatro significados parciales fundamentales correspondientes a las aportaciones de Leibniz, Cauchy, Fréchet y Robinson. En segundo lugar, se presenta la caracterización ontosemiótica de dichos significados a partir del análisis de la solución del problema de trazado de la tangente a una curva. Los resultados reflejan claras implicaciones, tanto para la enseñanza y aprendizaje en diversas carreras universitarias como para la formación de profesores de matemáticas.
\end{abstract}

Palabras clave: Diferencial. Significados. Configuración ontosemiótica. Análisis epistémico.

Manuel Alejandro Verón Doctorando en el programa de Doctorado en Ciencias Humanas y Sociales de la Universidad Nacional de Misiones (Argentina). Profesor (Universidad Nacional de Misiones), Posadas, Misiones, Argentina https://orcid.org/0000-0002-31601031 $\bowtie$ alejandroveron@fceqyn.unam.edu.ar

Belén Giacomone Doctorado en Ciencias de la Educación por la Universidad de Granada (España). Profesor asociado (Universidad de San Marino), Rep. de San Marino. (D) https://orcid.org/0000-0001-6752$\triangle \underline{\text { belen.giacomone@unirsm.sm }}$

Recebido em 10/05/2021 Aceito em 24/05/2021 Publicado em 22/06/2021

\section{Análise dos significados do conceito de diferencial de uma perspectiva ontosemiótica}

Resumo: Esta pesquisa aborda o estudo dos diversos significados do conceito de diferencial aplicando ferramentas teóricas do Enfoque Ontosemiótico, em particular as noções de significado pragmático de um conceito e configuração de práticas, objetos e processos. 0 objetivo é construir um modelo ontosemiótico de referência; para isso, em primeiro lugar, é realizado um estudo histórico-epistemológico sobre a origem e evolução do diferencial, identificando quatro significados parciais fundamentais correspondentes às contribuições de Leibniz, Cauchy, Fréchet e Robinson. Em segundo lugar, a caracterização ontosemiótica desses significados é apresentada a partir da análise da solução do problema de traçar a tangente a uma curva. Os resultados refletem implicações claras, tanto para o ensino e a aprendizagem nas várias carreiras universitárias, como para a formação de professores de matemática.

Palavras-chave: Diferencial. Significados. Configuração ontosemiótica. Análise epistêmica.

\section{Analyses of the meanings of the differential concept from a perspective onto-semiotic}

\begin{abstract}
This research addresses the study of various meanings of the differential concept applying theoretical tools of the Onto-Semiotic Approach, specifically the notions of pragmatic meaning of a concept, and configuration of practices, objects and process. The goal is to build a reference onto-semiotic model; for this, first, a historicalepistemological study is carried out on origin and evolution of the differential, identifying four fundamental partial meanings corresponding to Leibniz, Cauchy, Fréchet, and Robinson's contributions. Second, the onto-semiotic characterization about the meanings is presented from the analyses of the problem solution of calculating the tangent to a curve. Results show clear implications, both for teaching and learning in divers university careers and for mathematics teacher education.
\end{abstract}

Keywords: Differential. Meanings. Onto-semiotic configuration. Epistemic analyses. 


\section{Introducción}

El concepto de diferencial de una función es central en los currículos de diversas carreras universitarias. Las dificultades asociadas a este concepto no son nuevas y han sido reportadas por diversos autores (ARTIGUE, 1995; GÓMEZ, 2019; HU Y REBELLO, 2013; ORTON, 1983; TALL, 1981b); sin embargo, todavía representa un gran desafío para la educación universitaria y en consecuencia, una preocupación para la formación de profesores de matemáticas quienes deben afrontar la enseñanza en distintas disciplinas de ciencia, tecnología, ingeniería, física y matemáticas.

La literatura documenta que existen dificultades en torno al concepto de diferencial de tipo epistémico, cognitivo, pero también instruccional. Respecto a la dimensión epistémica, autores como Artigue, Menigoux y Viennot (1990), Bos (1974), Kleiner (2012), López-Gay (2001), Pulido (1997), Tall (1981a), Taylor (1974) y Valdivé y Garbin (2008) analizan la evolución histórica del concepto de diferencial y su estrecha relación con nociones como cantidad infinitamente pequeña, infinitesimal, aproximación lineal, aproximación o estimación lineal del incremento, destacando sus implicaciones en los procesos de enseñanza y aprendizaje.

Gómez (2019), Martínez-Torregrosa et al. (2002) y López-Gay et al. (2015) plantean que una de las dificultades de tipo cognitivo se produce por la diversidad de significados que se les presenta a los estudiantes, ya que hay que tener en cuenta las diferentes definiciones que se fueron presentando a lo largo del desarrollo del cálculo y su articulación con las diversas representaciones y contextos.

Las investigaciones mencionadas señalan diversas dificultades de tipo instruccional generadas, por un lado, por cómo se presenta el diferencial en los libros de textos de cálculo, antiguos y actuales, y, por otro lado, por el abordaje didáctico, que implica la gestión de la generalización y/o complejidad de conceptos según el significado que se pretenda trabajar con los estudiantes tanto de bachillerato como universitarios.

Para comprender y afrontar las distintas dificultades educativas, diversos autores han elaborados modelos parciales o clasificaciones de los significados del diferencial clarificando distintos usos y aplicaciones que hacen los estudiantes (DRAY y MANOGUE, 2010; HU y REBELLO, 2013; LÓPEZ-GAY et al., 2015; OLDENBURG, 2016; PULIDO, 2010, VALDIVÉ y GARBIN, 2008). Siguiendo esta línea, en este artículo se presenta un modelo de referencia sobre los significados del diferencial de una función, desde una perspectiva ontosemiótica (GODINO, BATANERO y FONT, 2007; 2020), dirigido a cursos preuniversitarios y primeros cursos de cálculo. Se espera que este modelo sea útil para realizar diferentes proyecciones en la enseñanza y aprendizaje de este concepto, e incidir en la formación de profesores de matemática en la didáctica del análisis matemático. Por ejemplo, en Lobo (2020) se observa la utilización 
del significado holístico de la derivada (PINO-FAN, GODINO y FONT, 2013) como marco de referencia para el diseño y evaluación de una propuesta de instrucción sobre el comportamiento del coronavirus covid-19.

Para la elaboración de dicho modelo, se toma como base los primeros resultados presentados por Verón (2020) recogidos de un estudio histórico epistemológico sobre origen y evolución del concepto de diferencial.

A continuación, en la sección 2, se describe el marco teórico y el problema específico de investigación. En la sección 3 se describe la metodología utilizada para la reconstrucción de los significados del concepto de diferencial. En la sección 4 se presentan los resultados obtenidos en función de las configuraciones ontosemióticas que caracterizan a los diversos significados, como así también sus interconexiones según niveles de generalización y formalización de los objetos intervinientes. Finalmente se presentan las conclusiones del estudio y sus implicaciones para la enseñanza y aprendizaje del cálculo.

\section{Marco teórico y problema de investigación}

\subsection{El enfoque Ontosemiótico del Conocimiento y la Instrucción Matemáticos (EOS)}

El enfoque Ontosemiótico del Conocimiento y la Instrucción Matemáticos (EOS) (FONT, GODINO y GALLARDO, 2013; GODINO, BATANERO y FONT, 2007) es un sistema teórico que adopta una visión antropológica y pragmatista de las matemáticas. Para la construcción del modelo de referencia de los significados del diferencial, se utilizan dos nociones claves de este enfoque: los significados sistémico-pragmático y configuración ontosemiótica de prácticas, objetos y procesos matemáticos.

\subsection{Significados sistémicos-pragmáticos}

Se define como práctica matemática a "toda actuación o expresión (verbal, gráfica, etc.) realizada por alguien para resolver problemas matemáticos, comunicar a otros la solución obtenida, validarla 0 generalizarla a distintos contextos y problemas" (GODINO y BATANERO, 1994, p. 334). Un objeto matemático, desde el punto de vista institucional se considera como un "emergente del sistema de prácticas sociales asociadas a un campo de problemas" (p. 335), y el significado de un objeto queda formado por el "sistema de prácticas institucionales asociadas al campo de problemas de las que emerge el objeto en un momento dado" (p. 338). 
Para identificar el significado pragmático de un objeto matemático, una estrategia metodológica consiste en seleccionar un problema prototípico, elaborar la secuencia de prácticas resolutivas e identificar la trama de objetos y procesos que se ponen en juego. Este análisis permite gestionar procesos de estudio y reconocer la complejidad ontosemiótica de la actividad matemática como fuente de explicación de las potenciales dificultades educativas (BURGOS y GODINO, 2020). El conjunto de los significados parciales de un objeto permite construir un significado de referencia del mismo, a partir de identificar las interconexiones entre ellos, lo que posibilita establecer niveles de generalización y formalización entre los significados.

\subsection{Configuración ontosemiótica de prácticas, objetos y procesos}

Para analizar el significado de los conceptos matemáticos se ha desarrollado la noción de configuración ontosemiótica de prácticas, objetos y procesos que intervienen en la resolución de situaciones-problemas, las cuales son la razón de ser de la actividad matemática y de los objetos emergentes de la misma. Los objetos matemáticos son clasificados en categorías según su naturaleza y función (GODINO et al., 2007, p. 132):

- lenguajes (términos, expresiones, notaciones, gráficos) en sus diversos registros (escrito, oral, gestual, etc.);

- situaciones-problemas (aplicaciones extra-matemáticas, ejercicios);

- conceptos-definiciones (introducidos mediante definiciones o descripciones);

— proposiciones (enunciados sobre conceptos);

— procedimientos (algoritmos, operaciones, técnicas de cálculo);

- argumentos (enunciados para justificar las proposiciones y procedimiento deductivos 0 de otro tipo).

En esta perspectiva, un objeto matemático es "cualquier entidad material o inmaterial que interviene en la práctica matemática, apoyando y regulando su realización" (GODINO et al., 2020, p. 6). Esta definición general de objeto se complementa con una tipología de objetos, dentro de la cual se incluyen los conceptos, entendidos desde una perspectiva unitaria como una entidad matemática que se define (concepto-definición). Pero sobre un concepto matemático, como el diferencial, se puede adoptar también una perspectiva sistémica, en cuyo caso el concepto de diferencial viene a ser la configuración ontosemiótica asociada a una de sus definiciones, y por tanto, al sistema de prácticas, objetos y procesos implicados en la resolución de un problema prototípico en que interviene el diferencial. 
Como para el concepto de diferencial, o cualquier otro concepto, se pueden encontrar diferentes definiciones ligadas a diferentes contextos de uso y situaciones-problemas, cada una de ellas constituye un significado parcial o sentido del concepto. El significado pragmático global del concepto de diferencial será, por tanto, la trama de configuraciones ontosemióticas que se puede reconstruir en las cuales dicho objeto interviene de manera clave.

\subsection{Problema de investigación}

Para la reconstrucción de los significados del concepto de diferencial de una función real de variable real partimos de los siguientes interrogantes:

1. ¿Cuáles son los diversos significados del diferencial?

2. ¿Qué elementos permiten distinguir los significados del diferencial?

3. ¿Cómo se relacionan y articulan entre si los diversos significados del diferencial?

4. ¿Es posible elaborar un modelo para categorizar los significados del diferencial según el grado de generalidad y formalización de los objetos intervinientes?

Esta reflexión permitirá caracterizar y establecer niveles entre los diversos significados del diferencial, en función de las configuraciones de prácticas, objetos y procesos, que intervienen en la resolución de las situaciones-problemas, donde se pone en juego el concepto de diferencial.

\section{Metodología}

La metodología es de tipo cualitativo descriptivo e histórico. La recolección de datos se basó en un estudio documental histórico-epistemológico sobre el origen y evolución del concepto diferencial para construir un significado de referencia del mismo. Se consultaron diferentes fuentes de información, como libros, revistas y páginas especializadas en didáctica de la matemática y en la historia de las matemáticas. Para la organización y descripción del análisis ontosemiótico, se optó por utilizar la tabla propuesta por Godino, Giacomone, Batanero y Font (2017, p. 104) cuya primera columna indica el enunciado y secuencia de prácticas elementales para resolver la tarea; los usos e intencionalidad de las mismas, en la segunda columna; y en la tercera columna, los objetos matemáticos que entran en juego en cada práctica. Luego de cada tabla realizamos una reflexión en torno a cómo entran en juego los objetos y procesos matemáticos identificados; esto es importante para reconocer el significado del concepto que está asociado a las prácticas matemáticas de la resolución del problema seleccionado. 
Existen diversas investigaciones en el marco del EOS que utilizaron la técnica del análisis ontosemiótico (Godino, 2017) para el estudio de los significados de diferentes contenidos matemáticos, como por ejemplo para el caso de la proporcionalidad (BURGOS y GODINO, 2020; GODINO et al., 2017), en el caso de los números naturales (GODINO et al., 2011), para las igualdad de números reales (WILHELMI, GODINO y LACASTA, 2007), para la derivada (PINO-FAN, GODINO y FONT, 2011) y la antiderivada (GORDILLO y PINO-FAN, 2016) y la integral definida (BURGOS et al., 2021).

\section{Análisis y resultados: significados parciales del concepto de diferencial}

La configuración de prácticas, objetos y procesos que se ponen en juego en la solución de un tipo de problema, en la que interviene de manera esencial el concepto de diferencial, constituye el significado pragmático parcial de dicho objeto matemático. No obstante, en dichas prácticas intervienen además otros objetos conceptuales, lingüísticos, procedimentales, proposicionales y argumentativos cuya identificaciones son importantes para reconstruir la trama de funciones semióticas en la que el concepto de diferencial participa.

El análisis de los significados del diferencial y sus relaciones con los conceptos fundamentales del Cálculo, como la derivada y la integral, lo vamos a realizar fijando la atención en los sistemas de prácticas que se realizan para resolver un problema específico en los marcos conceptuales elaborados por Leibniz, Cauchy, Fréchet y Robinson.

El problema específico que hemos elegido es el siguiente:

¿Cómo trazar la tangente a la parábola $y=x^{2}$ en un punto $P$ de la curva?

\subsection{Significado del diferencial en Leibniz}

En el Cálculo de Leibniz, una curva (cerrada) se concibe como un polígono de infinitos ángulos con un número infinito de lados de longitud infinitesimal, cada uno de los cuales coincide con una línea tangente a la curva. La secuencia básica de variables asociadas con la curva son las secuencias de abscisas $x$ y ordenadas $y$ de los infinitos vértices de este polígono.

La diferencia de dos valores sucesivos de $x$ es la diferencial $d x$, y similarmente para $d y$. Se supone que las cantidades $d x$ y $d y$ no son cero sino incomparablemente pequeñas, y por lo tanto despreciables, con respecto a los valores de las variables $x$ e $y$. Del mismo modo, se supone que un producto de los diferenciales, como $(d x)(d y) \circ(d x)^{2}$, es a su vez despreciable en comparación con los diferenciales $d x$ y $d y$. (EDWARDS, 1979, p. 261) 
Apoyándonos en las descripciones que hacen Bos (1974), Edwards (1979) y Pulido (1997), entre otros autores, de los conceptos y procedimientos que introdujo Leibniz para abordar los problemas del Cálculo, la solución del problema de la tangente (geométrica y algébrica) se puede abordar según la siguiente secuencia de prácticas discursivas y operativas.

Solución geométrica:

P1. Se considera a la curva $y=x^{2}$, junto con el eje de abscisas y la ordenada en $x=1$, como un polígono infinitoangular, es decir, un polígono con un número infinito de lados (Figura 1).

P2. "Encontrar una tangente es dibujar una línea recta que une dos puntos de la curva que tienen una distancia infinitamente pequeña, es decir, el lado prolongado del polígono infinitoangular que para nosotros es lo mismo que la curva" (LEIBNIZ, 1684, p. 223, citado en BOS, 1974, p. 19) (Figura 1)

Figura 1: La curva como línea infinito-poligonal

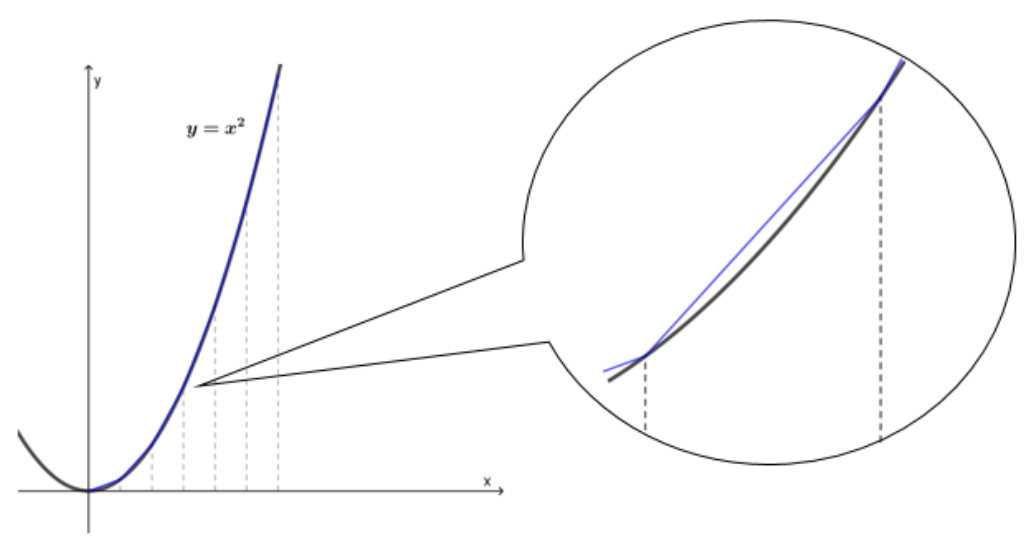

Fuente: Elaboración propia (2021)

P3. En la curva $y=x^{2}$ consideramos un lado $P Q$ del polígono infinitoangular, donde $P$ y $Q$ son dos puntos próximos cuya distancia es infinitamente pequeña. Al prolongar el lado $P Q$ se determinan nuevas cantidades geométricas variables como la subtangente $\sigma=A T$ y la tangente $\tau=P T$ (Figura 2). 
Figura 2: Prolongación del lado PQ para determinar la subtangente $\sigma=A T$

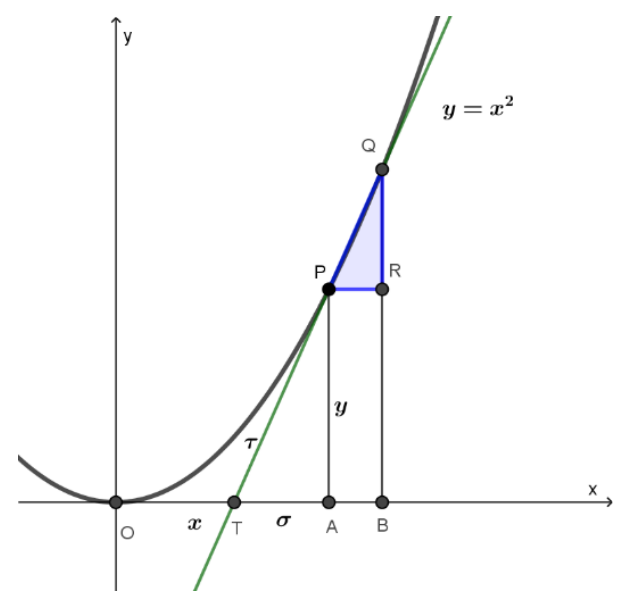

Fuente: Elaboración propia (2021)

P4. En el triángulo $P R Q$ los lados $P R, Q R$ y $P Q$ representan los incrementos o diferencias $\Delta x$, $\Delta y$ y $\Delta s$, respectivamente. $P Q$ es un lado del polígono infinitoangular cuya longitud es infinitamente pequeña. El triángulo $P R Q$ se denomina triángulo característico o diferencial de lados $d x, d y$ y $d s$ (Figura 3).

Figura 3: Triángulo diferencial de lados $\mathrm{dx}$, dy y ds

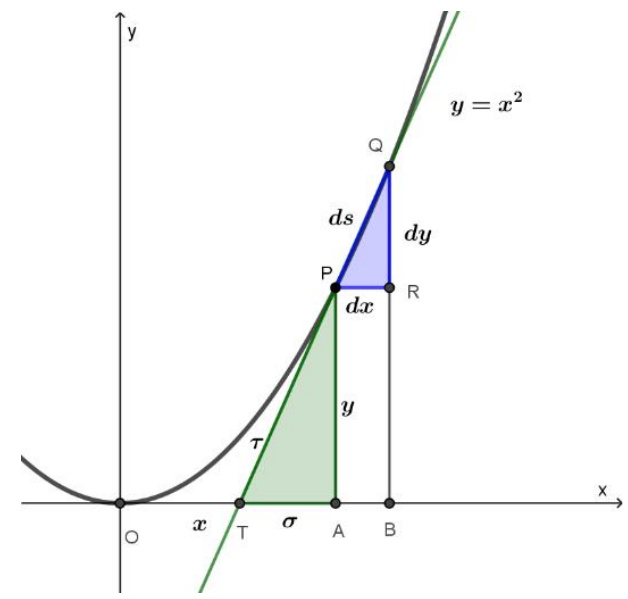

Fuente: Elaboración propia (2021)

P5. Los triángulos $P R Q$ (triángulo diferencial) y el $T A P$, determinado por la subtangente $\sigma$, la tangente $\tau$ y la ordenada $y$, son semejantes porque las hipotenusas están sobre la misma recta, los catetos correspondientes son paralelos y los ángulos correspondientes congruentes (Figura 3). Por tanto, las razones de los lados correspondientes son iguales: $d x: d y: d s=\sigma: y: \tau$, 
P6. $d y$ es el cuarto proporcional entre la subtangente, la ordenada y $d x$, es decir

(1) $\frac{d y}{d x}=\frac{y}{A T}$, siendo la subtangente $A T=\sigma$

P7. A partir de la ecuación (1) se obtiene $A T=y \frac{d x}{d y}$. Conocido AT es posible ubicar el punto T.

P8. La tangente a la curva por el punto $\mathrm{P}$ se obtiene trazando la recta que pasa por los puntos $\mathrm{P}$ y T.

Solución algebraica:

P9. Para el caso de la curva $y=x^{2}$, los puntos infinitamente próximos $\mathrm{P}$ y $\mathrm{Q}$ se relacionan de la siguiente manera $y+d y=(x+d x)^{2}$; esto es, $y+d y=x^{2}+2 x d x+(d x)^{2}$

P10. Como se observa en la Figura 3 la diferencia de las ordenadas de los puntos $Q$ y $R$ viene dada por $d y=(x+d x)^{2}-x^{2}$. Desarrollando el cuadrado del binomio y cancelando los términos $x^{2}$ resulta $d y=2 x d x+(d x)^{2}$. Luego se obtiene (2) $d y=2 x d x$, considerando que $(d x)^{2}=0$.

P11. De la expresión (2) se deduce que $d x=\frac{1}{2 x} d y$. Al multiplicar ambos miembros por $y, \mathrm{y}$ dividimos por $d y$, se obtiene: (3) $y \frac{d x}{d y}=\frac{y}{2 x}=\frac{x^{2}}{2 x}$

P12. El primer miembro de (3) representa la subtangente AT y el último, la expresión algebraica que permite calcular su valor en términos de cantidades conocidas y ubicar el punto $\mathrm{T}$.

P13. La recta tangente a la curva por el punto $P$ se obtiene trazando la recta que pasa por los puntos $\mathrm{P}$ y $\mathrm{T}$.

En el Cuadro 1 se incluye, para la secuencia de prácticas P1 a P8 indicadas anteriormente, el uso o intencionalidad de cada una de ellas en el proceso de resolución del problema, como así también los objetos matemáticos que se ponen en juego. Esta configuración constituye el significado sistémicopragmático del concepto de diferencial en el marco del cálculo infinitesimal de Leibniz, para el caso de la solución geométrica. El Cuadro 2 incluye la configuración correspondiente para la solución algebraica. 
Cuadro 1: Análisis ontosemiótico de la solución geométrica del problema de la tangente (en el marco del cálculo de Leibniz)

\begin{tabular}{|c|c|c|}
\hline $\begin{array}{l}\text { Secuencia de } \\
\text { prácticas } \\
\text { matemáticas } \\
\text { para resolver la } \\
\text { tarea }\end{array}$ & $\begin{array}{l}\text { Uso e intencionalidad de } \\
\text { las prácticas }\end{array}$ & $\begin{array}{l}\text { Objetos referidos en las prácticas (lenguaje, conceptos, } \\
\text { proposiciones, procedimientos, argumentos) }\end{array}$ \\
\hline P1 & $\begin{array}{c}\text { Visualizar la curva como un } \\
\text { polígono de infinitos lados. } \\
\text { Construir el "polígono } \\
\text { infinitoangular" }\end{array}$ & $\begin{array}{l}\text { Lenguajes: natural, geométrico-cartesiano, simbólico } \\
\text { Conceptos: curva, ecuación, eje de abscisas y ordenada, polígono } \\
\text { infinitoangular. } \\
\text { Procedimientos: trazar la poligonal sobre la curva considerando } \\
\text { puntos sucesivos cuya distancia es infinitamente pequeña. } \\
\text { Argumentos: la curva como línea infinito-poligonal }\end{array}$ \\
\hline P2 & $\begin{array}{c}\text { Explicitar cuál es el } \\
\text { procedimiento para hallar la } \\
\text { tangente a la curva en punto. }\end{array}$ & $\begin{array}{l}\text { Lenguaje: natural. } \\
\text { Conceptos: (...); tangente, línea recta, puntos infinitamente } \\
\text { próximos, magnitud longitud (distancia), prolongación. } \\
\text { Procedimientos: trazar una tangente es unir dos puntos sucesivos de } \\
\text { la curva con distancia infinitamente pequeña y prolongar el lado del } \\
\text { polígono infinitoangular } \\
\text { Argumentos: se aplica la definición de recta tangente a la curva. }\end{array}$ \\
\hline P3 & $\begin{array}{l}\text { Determinar los segmentos } \\
\text { tangente } \tau \text { y subtangente } \sigma \\
\text { a la curva en el punto P }\end{array}$ & $\begin{array}{l}\text { Lenguajes: (...); notaciones: } \sigma, \tau \text {, 'cantidades geométricas variables'. } \\
\text { Conceptos: (...); subtangente, cantidad geométrica variable. } \\
\text { Procedimientos: prolongar el lado PQ del polígono infinitoangular } \\
\text { para obtener la subtangente } \sigma=A T \text { y al tangente } \tau=P T \text {. } \\
\text { Argumentos: se aplica la definición de tangente y subtangente como } \\
\text { segmentos determinados por la recta tangente sobre los ejes. }\end{array}$ \\
\hline P4 & $\begin{array}{c}\text { Definir y representar el } \\
\text { triángulo característico o } \\
\text { diferencial de lados } d x, d y \text { y } \\
d s\end{array}$ & $\begin{array}{l}\text { Lenguajes: (...); notaciones: } \Delta x, \Delta y, \Delta s, d x, d y, d s \text { y 'triángulo } \\
\text { característico o diferencial'. } \\
\text { Conceptos: (...); triángulo diferencial, incrementos o diferencias, } \\
\text { diferenciales. } \\
\text { Procedimientos: Representación del triángulo diferencial. }\end{array}$ \\
\hline P5 & $\begin{array}{l}\text { Establecer la relación de } \\
\text { proporcionalidad entre los } \\
\text { lados del triángulo diferencial } \\
\text { y el triángulo formado por la } \\
\text { subtangente, ordenada y } \\
\text { tangente. }\end{array}$ & $\begin{array}{l}\text { Lenguaje: }(\ldots) ; \text { representación de igualdad de razones: } \\
d x: d y: d s=\sigma: y: \tau) \\
\text { Conceptos: }(\ldots) ; \text { triángulos semejantes, hipotenusa, catetos, } \\
\text { paralela, ángulos correspondientes, congruencia de ángulos, } \\
\text { razones y proporción. } \\
\text { Proposiciones: P1: "el triángulo diferencial y el triángulo TAP formado } \\
\text { por la subtangente, ordenada y tangente son semejantes"; } \\
\text { P2: "las razones entre los lados correspondientes de los triángulos } \\
\text { diferencial y TAP son iguales } d x: d y: d s=\sigma: y: \tau \text { " } \\
\text { Argumentos: relación de congruencia de ángulos y paralelismo de } \\
\text { lados. Corolario del Teorema de Tales. }\end{array}$ \\
\hline P6 & $\begin{array}{l}\text { Establecer la ecuación } \\
\text { proporcional } \\
\qquad \frac{d y}{d x}=\frac{y}{A T}\end{array}$ & $\begin{array}{l}\text { Lenguaje: (...). } \\
\text { Conceptos: (...); cuarto proporcional y ecuación proporcional. } \\
\text { Proposiciones: " } d y \text { es el cuarto proporcional entre la subtangente, } \\
\text { la ordenada y } d x " \\
\text { Procedimientos (conversión lenguaje natural a simbólico): } \frac{d y}{d x}=\frac{y}{A T} \\
\text { Argumentos: por la semejanza de triángulos. }\end{array}$ \\
\hline P7 & $\begin{array}{l}\text { Resolver la ecuación } \\
\frac{d y}{d x}=\frac{y}{A T} \text { para hallar la } \\
\text { subtangente AT }\end{array}$ & $\begin{array}{l}\text { Lenguaje: (...). } \\
\text { Conceptos: (...). } \\
\text { Proposiciones: } A T=y \frac{d x}{d y} \\
\text { Procedimientos: resolución de la ecuación proporcional. } \\
\text { Argumentos: propiedad fundamental de las proporciones. }\end{array}$ \\
\hline P8 & $\begin{array}{l}\text { Trazar la tangente PT a } \\
\text { partir de hallar la } \\
\text { subtangente AT }\end{array}$ & $\begin{array}{l}\text { Lenguaje: (...). } \\
\text { Conceptos: (...). } \\
\text { Proposiciones: "por los puntos P y T pasa la recta tangente a la curva } \\
\text { en el punto P". } \\
\text { Procedimientos: trazamos la recta tangente uniendo los puntos P y } \\
\text { T. }\end{array}$ \\
\hline
\end{tabular}


Argumentos: definición de tangente; por dos puntos paso una sola recta.

Fuente: Elaboración propia (2021)

En el sistema de prácticas P1 a P8 se llevan a cabo procesos de interpretación y significación de los diferentes términos y simbolizaciones que se usan, destacando el concepto de puntos infinitamente pequeños, cantidades geométricas variables, la curva como polígono infinitoangular, la tangente a la curva como recta que une dos puntos infinitamente próximos de la curva, empelando un lenguaje natural, geométrico y simbólico.

Como procesos de conceptualización destacan la definición de triángulo diferencial y los correspondientes elementos diferenciales $d x, d y$ y $d s$ como cantidades de longitud infinitamente pequeñas. Los diferenciales intervienen en procesos de algoritmización o cálculo una vez establecida la ecuación proporcional que relaciona las cantidades de magnitud geométrica, en este caso longitudes de segmentos.

Los argumentos que se utilizan provienen de la consideración de la curva como un polígono de infinitos lados, lo que permite determinar el triángulo diferencial y la semejanza con el triángulo formado por la subtangente, la ordenada y la tangente. A partir de establecer la semejanza entre los triángulos se establece las ecuaciones proporcionales que permiten hallar el valor de la subtangente AT y encontrar la tangente PT.

Cuadro 2: Análisis ontosemiótico de la solución algebraica del problema de la tangente (en el marco del cálculo de Leibniz)

\begin{tabular}{|c|c|c|}
\hline $\begin{array}{c}\text { Secuencia de } \\
\text { prácticas } \\
\text { matemáticas } \\
\text { para resolver la } \\
\text { tarea }\end{array}$ & $\begin{array}{l}\text { Uso e intencionalidad de } \\
\text { las prácticas }\end{array}$ & $\begin{array}{l}\text { Objetos referidos en las prácticas (lenguaje, conceptos, } \\
\text { proposiciones, procedimientos, argumentos) }\end{array}$ \\
\hline P9 & $\begin{array}{c}\text { Establecer la relación entre } \\
\text { los puntos } \mathrm{P} \text { y } \mathrm{Q}, \text { y la curva } \\
y=x^{2}\end{array}$ & $\begin{array}{l}\text { Lenguajes: algebraico, simbólico y expresiones: } x+d x, y+ \\
d y,(d x)^{2} \\
\text { Conceptos: curva, ecuación, puntos, longitud infinitesimal, } \\
\text { diferencial, diferencial de segundo orden. } \\
\text { Proposiciones: } \mathrm{P} \text { : "los puntos infinitamente próximos } \mathrm{P} \text { y Q se } \\
\text { relacionan de la siguiente manera } y+d y=(x+d x)^{2 "}, \mathrm{P} 2 \text { : "la } \\
\text { diferencia de la ecuación es } y+d y=x^{2}+2 x d x+(d x)^{2} \text { " } \\
\text { Procedimientos: se incrementan las variables } x \text { e } y \text { en una cantidad } \\
\text { infinitamente pequeña } d x \text { y } d y \text {, respectivamente; desarrollo del } \\
\text { binomio. } \\
\text { Argumentos: correspondencia entre las variables, donde un } \\
\text { incremento infinitesimal } d x \text { en } x \text { produce un incremento infinitesimal } \\
d y \text { en } y\end{array}$ \\
\hline P10 & $\begin{array}{l}\text { Determinar el } d y \text { como la } \\
\text { diferencia de las ordenadas } \\
\text { de los puntos } Q \text { y } R \text {. }\end{array}$ & $\begin{array}{l}\text { Lenguaje: (...). } \\
\text { Conceptos: (...); diferencia de ordenadas, cuadrado de un binomio, } \\
\text { propiedad cancelativa. } \\
\text { Proposiciones: } \mathrm{P} 1 \text { : "la diferencia de las ordenadas de los puntos } \mathrm{Q} \text { y } \\
\mathrm{R} \text { es } d y=(x+d x)^{2}-x^{2} ; \mathrm{P} 2 \text { : "los diferenciales de orden } \\
\text { superior se reemplazan por cero". }\end{array}$ \\
\hline
\end{tabular}




\begin{tabular}{|c|c|c|}
\hline & & $\begin{array}{l}\text { Procedimientos: plantear que el } d y \text { como la diferencia de las } \\
\text { ordenadas y luego de resolver el cuadrado del binomio; reemplazar } \\
(d x)^{2}=0 \\
\text { Argumentos: "La diferencia de dos valores sucesivos de } x \text { es la } \\
\text { diferencial } d x, y \text { similarmente para } d y ;(d x)^{2} \text { es despreciable en } \\
\text { comparación con los diferenciales } d x \text { y } d y \text { " (EDWARDS, 1979, p. } \\
261)\end{array}$ \\
\hline P11 & $\begin{array}{l}\text { Manipular algebraicamente } \\
\text { la ecuación } \\
d y=2 x d x \text { para escribirla } \\
\text { como } y \frac{d x}{d y} \text { (expresión de la } \\
\text { subtangente AT) }\end{array}$ & $\begin{array}{l}\text { Lenguajes: (...); expresiones: } y \frac{d x}{d y} \\
\text { Conceptos: (...); segmento y subtangente. } \\
\text { Procedimientos: primero se despeja } d x \text {, luego se multiplica por } y \text { y } \\
\text { se divide por } d y \text { para obtener la expresión } y \frac{d x}{d y} \text { Por último se } \\
\text { reemplaza la ecuación de la curva. } \\
\text { Argumentos: multiplicación y división a ambos miembros de la } \\
\text { ecuación. } y \frac{d x}{d y} \text { es la expresión de la subtangente }\end{array}$ \\
\hline P12 & $\begin{array}{l}\text { Reconocer la expresión de la } \\
\text { subtangente }\end{array}$ & $\begin{array}{l}\text { Lenguaje: natural. } \\
\text { Conceptos: (...). } \\
\text { Proposiciones: "la expresión de la subtangente es } y \frac{d x}{d y} \text { " } \\
\text { Procedimientos: calcular el valor de la subtangente AT para ubicar } \\
\text { el punto T. }\end{array}$ \\
\hline P13 & Trazar la recta tangente & $\begin{array}{l}\text { Lenguaje: geométrico. } \\
\text { Conceptos: (...); recta y tangente. } \\
\text { Proposiciones: enunciado de la P13 } \\
\text { Procedimientos: trazado de la recta que pasa por dos puntos. } \\
\text { Argumentos: secuencia de prácticas P9 a P12. }\end{array}$ \\
\hline
\end{tabular}

Fuente: Elaboración propia (2021)

En el sistema de prácticas, realizadas en la solución algebraica, se destaca el papel algorítmico desempeñado por el lenguaje algebraico; los elementos diferenciales $d x, d y$ intervienen como cantidades infinitesimales como si fueran datos conocidos con los que se puede operar.

Se destaca en P9 el procedimiento de diferencia de la ecuación en el cual intervienen una proposición fundamental en el cálculo de Leibniz "un incremento infinitesimal $d x$ en $x$, produce un incremento infinitesimal $d y$ en $y$ " (MARTíNEZ-TORREGROSA et al., 2002). En P10 se asume como verdadera otra proposición fundamental, P2: "Las diferencias de orden superior se reemplazan por cero". Estas proposiciones junto con el aparato algebraico desarrollado por Leibniz que se presentan en los procedimientos, caracterizan la manera en el cual eran utilizados los diferenciales.

Los procedimientos algebraicos utilizados para hallar la expresión de la subtangente AT como $y \frac{d x}{d y}$ permiten identificar la trama de objetos y procesos que intervienen para determinar la tangente PT a la curva en el punto $P$.

Observamos en los sistemas de prácticas matemáticas en el marco del cálculo de Leibniz, la utilización de un lenguaje natural, geométrico, algebraico y simbólico, siendo este último, una de sus principales características por su persistencia en el tiempo (BOS, 1974; EDWARDS, 1979; KLEINER, 2012; MARTíNEZ-TORREGROSA et al., 2006; PULIDO, 1997). 
El lenguaje geométrico se caracteriza por la utilización de los conceptos como cantidades geométricas variables, es decir, considerar a las variables como cantidades de magnitud. La utilización del triángulo diferencial para el cálculo de la subtangente y la tangente a una curva, la cual era considerada como un polígono de infinitos lados infinitamente pequeño.

El lenguaje algebraico se caracteriza por el procedimiento del cálculo de la subtangente y la tangente por medio de la diferencia de la ecuación de la curva, considerando que los diferenciales de orden dos son insignificantes o despreciables en comparación con $d x$ y $d y$ (BOS, 1974; EDWARDS, 1979). La simbología introducida para denotar las cantidades infinitamente pequeñas o infinitesimales son $d x$ y $d y$, para las variables $x$ e $y$, respectivamente; las cuales continúan siendo utilizadas en la actualidad.

Los sistemas de prácticas de la solución geométrica y algebraica permiten identificar un significado del concepto de diferencial, el diferencial de Leibniz, a partir del análisis ontosemiótico realizado identificando los objetos que intervienen y emergen en la resolución de la situación-problema del cálculo de la tangente a una curva en un punto.

\subsection{Significado del diferencial en Cauchy}

Cauchy definió la diferencial como una expresión construida a partir de la derivada: $d f=$ $f^{\prime}(x) \cdot d x$, siendo $d x$ un incremento arbitrario de la variable y pasó a convertirse así en un simple instrumento formal, necesario para justificar y abreviar ciertas demostraciones. Se desprendió, entonces, a la diferencial de la ambigüedad de los infinitamente pequeños, pero al mismo tiempo quedó desprovista de cualquier significado físico o intuitivo propio: simplemente era el producto de la derivada por el incremento de la variable independiente (MARTÍNEZ-TORREGROSA et al., 2002).

Se incluye, a continuación, la definición que aparece en Cauchy (1823, p. 13):

Sea $y=f(x)$ una función de la variable independiente $x, i$ una cantidad infinitamente pequeña, $y$ $h$ una cuantidad finita. Si se plantea que $i=\alpha h, \alpha$ será todavía una cantidad infinitamente pequeña, y se tendrá idénticamente

De donde se concluirá

$$
\frac{f(x+i)-f(x)}{i}=\frac{f(x+\alpha h)-f(x)}{\alpha h}
$$

(1) $\frac{f(x+\alpha h)-f(x)}{\alpha}=\frac{f(x+i)-f(x)}{i} h$

El límite hacia el cual converge el primer miembro de la ecuación (1), cuando la variable $\alpha$ se aproxima indefinidamente a cero y la cantidad $h$ permanece constante, es lo que se llama la diferencial de la función $y=f(x)$. Esta diferencial se indica por la característica $d$, de donde se sigue: $d y$ o $d f(x)$. Es fácil obtener su valor cuando se conoce el de la función derivada $y^{\prime}$ o $f^{\prime}(x)$. En efecto, tomando los límites en los dos miembros de la ecuación (1) se encuentra

(2) $d f(x)=h f^{\prime}(x)$. En el caso particular donde $f(x)=x$, la ecuación (2) se reduce a

(3) $d x=h$. 
Así, la diferencial de la variable independiente $x$ no es otra cosa que la constante finita $h$. Por tanto, la ecuación (2) viene a ser

(4) $d f(x)=f^{\prime}(x) \cdot d x$.

o lo que viene a ser lo mismo,

(5) $d y=y^{\prime} d x$.

El cálculo de Cauchy se caracteriza principalmente por la consideración de las cantidades como variables, a diferencia de Leibniz que consideraba a las cantidades geométricas variables como una cantidad infinitamente pequeña, menor que cualquier cantidad finita pero no nula (KLEINER, 2012). Para Cauchy (1821) "una cantidad variable se vuelve infinitamente pequeña, cuando su valor numérico disminuye indefinidamente para converger hacia el límite cero" (p. 26). Una cantidad infinitamente pequeña, denotada por $\alpha \circ i$, es "una variable cuyo valor numérico disminuye indefinidamente" (CAUCHY, 1821, p. 27).

En Cauchy (1823, p. 23-24) encontramos la siguiente explicación del problema de la tangente a una curva, que describe como determinar la inclinación de una curva en un punto. Para hacer un análisis de los tipos de objetos y procesos que pone en juego en la solución del problema dividimos la explicación dada por Cauchy en prácticas elementales:

P1. Consideremos la curva que tiene por ecuación en coordenadas rectangulares $y=f(x)$.

P2. En esta curva, la cuerda trazada desde el punto $(x, y)$ al punto $(x+\Delta x, y+\Delta y)$, forma, con el eje de las $x$ prolongado en el sentido de las $x$ positivas, dos ángulos, uno agudo, el otro obtuso, donde el primero mide la inclinación de la cuerda, con respecto al eje de las $x$.

P3. Si el segundo punto se aproxima una distancia infinitamente pequeña del primero, la cuerda se confundirá sensiblemente con la tangente trazada a la curva por el primer punto;

P4. y la inclinación de la cuerda, con relación al eje de las $x$, vendrá a ser la inclinación de la tangente, o lo que se llama la inclinación de la curva respecto al mismo eje.

P5. Planteado así, como la inclinación de la cuerda tendrá por tangente trigonométrica el valor numérico de la razón $\frac{\Delta y}{\Delta x}$, es claro que la inclinación de la curva tendrá por tangente trigonométrica el valor numérico del límite hacia el cual converge esta razón, es decir, de la función derivada $y^{\prime}=\frac{d y}{d x}$.

P6. Si el valor de $y^{\prime}$ es nulo o infinito, la tangente a la curva será paralela o perpendicular al eje de las $x$. Cuando es paralela, la ordenada y viene a ser un máximo o un mínimo. 
A continuación, se describe, a modo de ejemplo, algunos puntos importantes que observamos en la configuración de prácticas y objetos que constituye el significado sistémico-pragmático del concepto de diferencial en el marco del Cálculo de Cauchy.

En el sistema de prácticas desarrolladas en este marco, se observa que el lenguaje empleado es geométrico, algebraico y funcional, donde se emplean conceptos y términos, como inclinación, cuerda, curva, tangente, infinitamente pequeño, tangente trigonométrica, límite, convergencia, razón de incrementos, diferenciales, cociente de diferenciales y función derivada. Estos conceptos son utilizados en el proceso de definición de la inclinación de la tangente y de la curva a partir de la inclinación de la cuerda, cuando los puntos que la definen se aproximan infinitamente y la distancia que los separa es una variable que tiene por límite cero.

Como proposición se plantea que la inclinación de la cuerda es medida mediante la razón entre incrementos, denominado tangente trigonométrica; y como procedimiento de cálculo se realiza el proceso de paso al límite que da lugar a la derivada de la función en el punto $(x, y)$. Los diferenciales aparecen como mera representación alternativa de la derivada. Los argumentos que validan los procedimientos y proposiciones son la definición de inclinación de la curva, la tangente trigonométrica como la razón de los incrementos, el límite y la consideración de las cantidades infinitamente pequeñas como una variable cuyo límite es cero, la función derivada como límite de la razón de incremento, y la igualdad entre la función derivada y el cociente de diferenciales.

El concepto de diferencial pasa a un segundo plano con Cauchy. Esto es debido a que la introducción de los límites permitió el paso de la razón de incrementos a la función derivada, colocando al cociente de diferenciales como expresión equivalente a la derivada. Donde antes eran necesario los diferenciales para hallar la tangente, ahora ya no lo son.

\subsection{Significado del diferencial en Fréchet}

Según Artigue (1989, p. 34), el diferencial fue definido como una función lineal, por primera vez por M. Fréchet en 1911, en el marco del desarrollo del análisis funcional. De hecho, la definición que introduce de diferencial rehabilita la vieja idea de aproximación que había predominado al comienzo del cálculo, pero había sido puesta a un lado después por razones de falta de rigor. La idea de función lineal y aproximación queda patente en la definición de diferencial de Fréchet:

Una función $f(x, y, z, t)$ admite una diferencial en el punto $\left(x_{0}, y_{0}, z_{0}, t_{0}\right)$ si existe una función lineal y homogénea de los incrementos, o sea: $A \Delta x+B \Delta y+C \Delta z+D \Delta t$, que no difiere del incremento de la función $\Delta f$ a partir del valor $f\left(x_{0}, y_{0}, z_{0}, t_{0}\right)$ nada más que en un 
valor infinitamente pequeño respecto a la distancia $\Delta$ de los puntos $\left(x_{0}, \ldots, t_{0}\right)$ y $\left(x_{0}+\right.$ $\left.\Delta x \ldots, t_{0}+\Delta t\right)$. La diferencial entonces es por definición: $d f=A \Delta x+B \Delta y+C \Delta z+$ $D \Delta t . "$

Esta definición se expresa por la fórmula $\Delta f=d f+\varepsilon \Delta$ donde $\varepsilon$ tiende a cero cuando $\Delta$ tiende a cero. (ARTIGUE, 1989, p. 34)

La definición de Fréchet no requiere que el diferencial sea infinitamente pequeño, sino que $\Delta f-$ $d f$ es infinitamente pequeño respecto $\Delta$; esto no significa que $(\Delta f-d f)$ será siempre un número muy pequeño, o incluso menos que $\Delta f$ odf sean pequeños. El requisito que se plantea es que $(\Delta f-d f)$ tienda a cero más rápidamente que $\Delta$, esto es que el límite de $(\Delta f-d f) / \Delta$ es cero cuando $\Delta$ tiende a cero. Esto quiere decir que $d f$ es una función lineal homogénea de los incrementos, y se puede expresar al $\Delta f$ de la siguiente manera: $d f+\varepsilon \cdot \Delta$, donde $\lim _{\Delta \rightarrow 0} \varepsilon=0$ (MARTíNEZ-TORREGROSA et al., 2006, p. 456).

De esta manera, el diferencial de Fréchet se define como la estimación lineal del incremento respecto al cambio de variable. Se entiende que el diferencial es una función que puede tomar cualquier valor y su expresión diferencial va a estar sujeta a la condición de que $(\Delta f-d f)$ sea infinitamente pequeña respecto a $\Delta x$. Esto quiere decir que $d f$ no es infinitamente pequeño, sino que la diferencia entre el incremento y el diferencial es infinitamente pequeño respecto a $\Delta x$ (LÓPEZ-GAY, 2001).

Sabiendo que el diferencial se define como una aplicación lineal, resulta necesario destacar que $d f$ es una función de dos variables (MARTíNEZ-TORREGROSA et al., 2002) ya que depende del punto $x=a$ y del diferencial de $x$, cuya notación es: $d f(a, d x)=f^{\prime}(a) d x$

Problema: Hallar una aplicación lineal homogénea que aproxima a la función $y=x^{2}$ en el punto $a$ de tal modo que el error que se comete en la estimación, relativo al incremento $\Delta x$ de la variable $x$, es infinitamente pequeño respecto de dicho incremento.

\section{Solución:}

P1. Dada la función $f: A \rightarrow R$ tal que $f(x)=x^{2}$, se busca una aplicación lineal y homogénea que aproxime $f(x)=x^{2}$ en el punto $a$, de tal manera que el error que se produce con la estimación respecto al incremento $\Delta x$ de la variable $x$ sea infinitamente pequeño respecto del incremento $\Delta x$.

P2. El incremento de la función $f(x)=x^{2}$ en el punto $a$ respecto del incremento $\Delta x$ es $\Delta f=$ $f(a+\Delta x)-f(a)=(a+\Delta x)^{2}-a^{2}$

P3. Luego tenemos que $\Delta f=f(a+\Delta x)-f(a)=f^{\prime}(a) d x+\varepsilon d x$ con $f^{\prime}(a)=2 a$. 
P4. Entonces, si $\varepsilon$ tiende a cero cuando $\Delta x$ tiende a cero, el $\Delta f \approx f^{\prime}(a) d x$, es decir $\Delta f \approx$ $2 a d x$ en el punto $a$.

P5. La aplicación lineal y homogénea que aproxima al incremento de la función en el punto $a$ respecto al incremento $\Delta x$ es la función $d f(a)=2 a d x$

El sistema de prácticas matemáticas de P1 a P5 permiten caracterizar el significado del diferencial de Fréchet, donde se utilizan los lenguajes natural, geométrico, algebraico y simbólico. Los conceptos y términos que emergen de las prácticas son: aplicación lineal y homogénea, función, variable, incremento, aproximación, estimación, error, infinitamente pequeño, límite y derivada.

Los enunciados y proposiciones que se realizan sobre los conceptos y definiciones son: " $\Delta f=$ $f^{\prime}(a) d x+\varepsilon d x$ " (el incremento de la función se puede escribir como una suma de una aplicación lineal y homogénea del incremento de $x$ y una función $\varepsilon$ que tiende a cero cuando $\Delta x$ tiende a cero), y " $d f=$ $f^{\prime}(a) d x$ es una aplicación lineal y homogénea del incremento de $x^{\prime \prime}$.

Los procedimientos que se emplean para determinar la aplicación lineal y homogénea que aproxime al incremento de la función provienen del cálculo de límites y derivadas. En cuanto a los argumentos que validan los procedimientos y proposiciones se utilizan la definición de función, límite, incremento de la función $\Delta f=d f+\varepsilon \Delta$ y el diferencial como una aplicación lineal y homogénea del incremento de $x$.

\subsection{Significado del diferencial en el análisis no estándar}

El análisis no-estándar desarrollado por Robinson (1966) mostró que es posible definir los conceptos fundamentales del análisis (continuidad, diferenciación, integración, etc.) en términos de infinitesimales, en lugar de tener que usar necesariamente el concepto de límite, lo cual conecta esta teoría matemática con la aproximación histórica al cálculo infinitesimal de Leibniz.

Una idea central es construir una ampliación del conjunto $\mathrm{R}$ de los números reales a ${ }^{*} \mathrm{R}$ (conjunto de números hiperreales) que incluye como nuevos elementos números infinitamente pequeños e infinitamente grandes, considerados como números hiperreales. Como estructura algebraica es un cuerpo no arquimediano y métricamente incompleto que contiene al conjunto arquimediano y completo identificable con los números reales.

Robinson (1966 p. 56) define de la siguiente manera los números hiperreales infinitésimos e infinitos: 
Un número $a \epsilon^{*} R$ se llamará infinitesimal o infinitamente pequeño si $|a|<m$ para todo número positivo $m \in \mathrm{R}$. Según esta definición, 0 es infinitesimal. ...Un número $r \epsilon^{\star} \mathrm{R}, r \neq 0$, es infinitesimal si y solo si $r^{-1}$ es infinito. Si $a-b$ es infinitesimal, entonces decimos que $b$ está infinitamente próximo a $a$, y se escribe $a \simeq b$. Esto es, dos hiperreales $x, y \epsilon^{*} \mathrm{R}$ son infinitamente cercanos (notación: $x \simeq y$ ), $x \simeq y \quad \Leftrightarrow \quad(\forall n \in \mathbb{N}, n \geq 1)|x-y|<1 / n$

El cuerpo *R proporciona un marco para el desarrollo del cálculo diferencial e integral mediante los números hiperreales infinitamente pequeños e infinitamente grandes.

Obviamente, el uso de los infinitesimales en el análisis no-estándar recuerda al cálculo infinitesimal de Leibniz, y el análisis no-estándar se puede considerar por los matemáticos actuales como una rehabilitación del uso de las cantidades infinitamente pequeñas de Leibniz. Esta visión es fuertemente defendida por Robinson. (BOS, 1974, p. 82)

No obstante, Bos (1974) no comparte plenamente la opinión de Robinson. Para probar su valor como una teoría matemática el cálculo de Leibniz no necesita un ajuste a los requisitos de aceptabilidad de las matemáticas del siglo XX mediante una reformulación en términos del análisis no-estándar. Considera, además que ambas teorías difieren sustancialmente. Un aspecto fundamental en que ambas teorías difieren se refiere a la concepción del conjunto de infinitesimales. Leibniz y la mayoría de sus seguidores concibieron que el conjunto de infinitesimales estaba constituido de sucesivos órdenes de pequeñez infinita. Así, si $d x$ era un diferencial de primer orden, entonces todos los diferenciales de primer orden tienen una razón finita con $d x$; en general todos los diferenciales de orden $n$ están en razón finita con $d x^{n}$, y el conjunto de los infinitesimales está formado sólo de estas clases de diferenciales.

Otra diferencia entre ambas teorías está en el hecho de que el análisis infinitesimal de Leibniz trata con cantidades geométricas, variables y diferenciales, mientras que el análisis no-estándar, así como el análisis real moderno en general, trata con números reales, funciones y derivadas, a pesar de la aceptación de los diferenciales. Los problemas relacionados con la diferenciación de órdenes superiores de cantidades variables no ocurren en el análisis no estándar.

Keisler (2000, p. 23) usa el problema de la tangente a la parábola $y=x^{2}$ para explicar de manera intuitiva las dificultades de usar las cantidades infinitamente pequeñas $\Delta y, \Delta x$ para calcular la pendiente a una curva (Figura 4). La pendiente promedio para un incremento $\Delta x$ viene dada por, $\Delta y / \Delta x=2 x_{0}+\Delta x, y$ por tanto solo se puede calcular cuando $\Delta x$ es distinto de cero porque de lo contrario el cociente $\Delta y / \Delta x$ está indefinido. 
Figura 4: Relación entre la pendiente y los incrementos

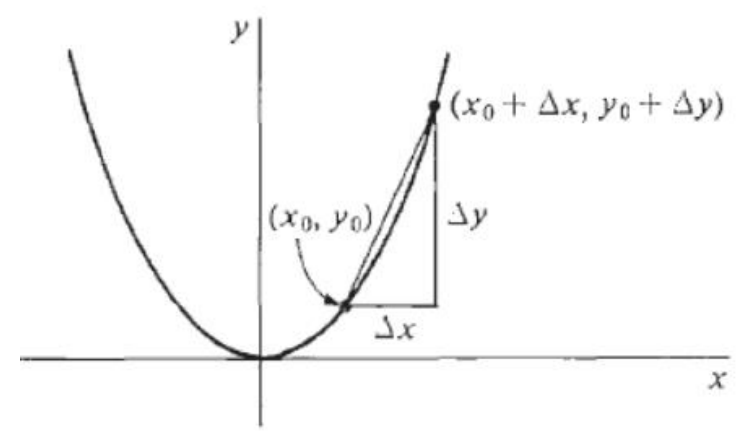

Fuente: KEISLER (2000, p. 26)

El razonamiento en el cálculo de Leibniz es que tomando $\Delta x$ infinitamente pequeño aunque no nulo entonces el término $\Delta x$ se puede despreciar y de este modo se tiene que $\frac{\Delta y}{\Delta x}=2 x_{0}$

Se necesita establecer una distinción nítida entre los números que son suficientemente pequeños para ser despreciables en los cálculos y los que no lo son. De hecho, ningún número real excepto el cero es suficientemente pequeño como para ser despreciable. El análisis no estándar resuelve este problema al crear los números hiperreales, y dentro de ellos números infinitesimales o infinitamente pequeños.

En la siguiente Figura 5 se muestra la distinciòn entre incremento y diferencial.

Figura 5: Distinción entre incremento y diferencial

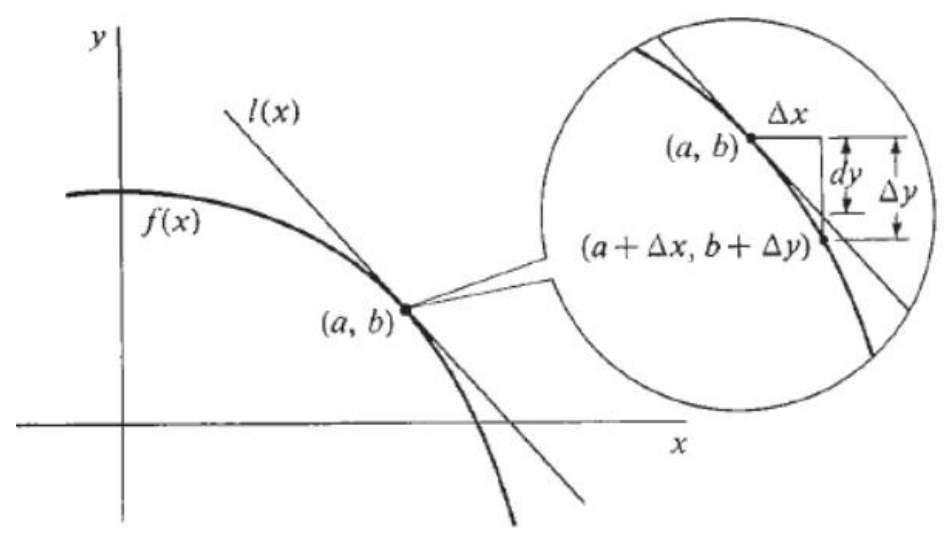

Fuente: KEISLER (2000, p. 56)

Supongamos que $y$ depende de $x, y=f(x)$ :

(i) La diferencial de $x$ es la variable independiente $d x=\Delta x$.

(ii) El diferencia de $y$ es la variable dependiente $d y$ dada por $d y=f^{\prime}(x) d x$.

La relación entre la derivada y el cociente de diferenciales es la igualdad: $\frac{d y}{d x}=f^{\prime}(x)$ 


\section{revemop}

Mientras que la derivada y el cociente incremental difieren en un infinitésimo:

$$
\frac{\Delta y}{\Delta x} \approx f^{\prime}(x)
$$

Veamos cómo se aborda el cálculo de la pendiente a la curva $y=x^{2}$ en el punto $\left(x_{0}, y_{0}\right)$ usando los números hiperreales infinitésimos.

Problema de la tangente:

P1. Considera un punto real $\left(x_{0}, y_{0}\right)$ sobre la curva $y=x^{2}$. Sea $\Delta x$ un infinitésimo positivo 0 negativo (pero no cero), y $\Delta y$ el cambio correspondiente en $y$.

P2. La pendiente en $\left(x_{0}, y_{0}\right)$ se define del siguiente modo:

[pendiente en $\left.\left(x_{0}, y_{0}\right)\right]=\left[\right.$ el número real infinitamente próximo a $\left.\frac{\Delta y}{\Delta x}\right]$

P3. $\frac{\Delta y}{\Delta x}$ se calcula de igual modo que se ha hecho antes

$$
\frac{\Delta y}{\Delta x}=\frac{\left(x_{0}+\Delta x\right)^{2}-x_{0}^{2}}{\Delta x}=2 x_{0}+\Delta x
$$

P4. Este es un número hiperreal, no uno real. Puesto que $\Delta x$ es infinitesimal, el número hiperreal $2 x_{0}+\Delta x$ está infinitamente próximo al número real $2 x_{0}$.

P5. Concluimos que [pendiente en $\left.\left(x_{0}, y_{0}\right)\right]=2 x_{0}$

El mismo método se puede aplicar a cualquier otra curva.

El significado sistemico-pragmático del diferencial en el marco del análisis no estándar de Robinson se caracteriza por los objetos primarios que intervienen en los procesos de definición y cálculo de la pendiente a una función $y=f(x)$ donde se utiliza el lenguaje natural, geométrico, algebraico y simbólico. Los conceptos que entran en juego en las prácticas son: infinitésimo, número hiperreal, número real, números infinitamente próximos, curva, pendiente, y razón de incrementos infinitesimales. Estos conceptos son empleados para establecer proposiciones como: "El número hiperreal $2 x_{0}+\Delta x$ no es un número real"; "El número hiperreal $2 x_{0}+\Delta x$ está infinitamente próximo al número real $2 x_{0}$ "

En cuanto a los procedimientos se observan que se plantea la razón de incrementos infinitesimales y que se resuelve mediante el cálculo algebraico con números hiperreales. Es por ello que los argumentos que validan esta resolución provienen de la definición de números hiperreales y de las propiedades algebraicas de las operaciones con números hiperreales. 
En el sistema de prácticas descritas en el marco del análisis no estándar se puede identificar un significado del diferencial, el diferencial de Robinson en el análisis no estándar, el cual se caracteriza por las configuraciones de prácticas y objetos de donde surge el concepto.

\subsection{Síntesis y estructuración de los significados del concepto de diferencial}

El análisis ontosemiótico realizado sobre cada significado caracterizado por la configuración de prácticas, objetos y procesos, permiten establecer interconexiones entre los significados parciales y configurar un significado de referencia (GODINO et al., 2017).

Las configuraciones ontosemióticas (CO) las hemos denominado de la siguiente manera:

- CO1: Diferencial de Leibniz,

- $\mathrm{CO}_{2}$ : Diferencial de Cauchy,

- $\mathrm{CO}_{3}$ : Diferencial de Fréchet y

— CO4: Diferencial en el análisis no estándar.

Resulta interesante realizar diferentes observaciones identificando algunas interconexiones entre las configuraciones. En primer lugar, podemos destacar que a lo largo de la evolución histórica del concepto diferencial, la situación-problema que hemos seleccionado se mantiene en gran parte, comenzando en Leibniz con el cálculo de la tangente, luego pasando al cálculo de la inclinación de la curva con Cauchy, cálculo de la aplicación lineal que aproxima al incremento de la función con Fréchet y finalmente el cálculo de la pendiente en el análisis no estándar. Esto quiere decir, que la resolución de un mismo problema se puede abordar en los diferentes marcos conceptuales considerados, pero los objetos que intervienen van cambiando. Esta última característica que hemos mencionado responde al propio desarrollo histórico de las matemáticas, y es una particularidad de la historia del cálculo, el avance del cálculo tuvo varias etapas: ingenuo, formal y crítico (KLEINER, 2001).

En la resolución del problema emerge un primer objeto a estudiar, que son los conceptosdefiniciones que en el diferencial de Leibniz se caracteriza principalmente por el uso de las cantidades infinitamente pequeñas, los infinitesimales, para obtener la subtangente y la tangente. Con el diferencial de Cauchy, los infinitesimales se reconfiguran y pasan a ser considerados como variables que tienen límite cero cuando el incremento de $x$ tiende a cero. A partir de los conceptos de límite, convergencia, tangente trigonométrica y derivada, el diferencial pasa de ocupar un lugar central en el cálculo (BOYER, 1987; EDWARDS, 1979; KLEINER, 2012), a un lugar marginal definiéndose como una expresión que depende de la derivada (MARTÍNEZ-TORREGROSA et al., 2002). 
Luego, en el diferencial de Fréchet, los infinitesimales siguen apareciendo como un objeto necesario para el surgimiento de los nuevos conceptos que emergen de las prácticas matemáticas, como la aplicación lineal y homogénea, aproximación lineal y la estimación. Finalmente, los infinitesimales que surgieron con Leibniz, en el siglo XX son definidos formalmente en el análisis no estándar con Robinson (1966), donde entran en juego nuevos conceptos entorno a la $\mathrm{CO}_{4}$, como números hiperreales y números infinitamente próximos, pero muchos otros se siguen manteniendo como pendiente, razón de incrementos infinitesimales, etc. Los conceptos que intervienen en la $\mathrm{CO}_{4}$ en el marco del análisis no estándar permiten identificar un cierto nivel de generalización y formalización, producto del propio avance de las matemáticas.

En la evolución de los lenguajes, se observa que el diferencial está íntimamente relacionado al geométrico y natural, aunque también se destacan los lenguajes algebraicos y simbólicos con la introducción de $d x$, dy en la $\mathrm{CO}_{1}$. A medida que fueron surgiendo las otras $\mathrm{CO}$, adquirieron relevancia otros lenguajes, por ejemplo, en la $\mathrm{CO}_{2}$ con Cauchy, se destaca el algebraico y simbólico, principalmente por la expresión $d f=f^{\prime}(x) d x$; en la $\mathrm{CO}_{3}$, con Fréchet, el lenguaje algebraico, funcional y simbólico al trabajar con las aplicaciones lineales y la aproximación lineal del incremento de $f$; en el análisis no estándar se destaca el geométrico, algebraico y simbólico con la formalización de los infinitesimales.

En cuanto a las proposiciones, es interesante resaltar cómo se enuncian y relacionan los conceptos de cada CO para enunciar diferentes afirmaciones sobre el diferencial, donde algunas tienen puntos en común y otras no. Los procedimientos en cada $\mathrm{CO}$ tienen puntos de encuentro, por ejemplo, en el planteo de la tangente trigonométrica en la $\mathrm{CO}_{2}$ con Cauchy y la razón de incrementos infinitesimales en el análisis no estándar $\left(\mathrm{CO}_{4}\right)$, aunque cada procedimiento tiene asociado una $\mathrm{CO}$ que permite construir un significado sistémico-pragmático parcial del diferencial. Los argumentos que se emplean para validar los enunciados y razonamientos están en estrecha relación con los conceptosdefiniciones involucrados en cada $\mathrm{CO}$.

En general, se observa que las $\mathrm{CO}$ asociadas al diferencial presentan varias interconexiones las cuales permiten establecer niveles de generalización y formalización entre ellas, a partir de las prácticas matemáticas (operativas y discursivas) y los objetos que intervienen y emergen de las mismas. Como primer nivel colocamos a la $\mathrm{CO}_{1}$ : diferencial de Leibniz ya que se considera el origen del diferencial asociado principalmente al concepto-definición de las "cantidades infinitamente pequeñas". En el segundo nivel, colocamos a la $\mathrm{CO}_{2}$ : diferencial de Cauchy porque hay una evolución en los objetos que intervienen y destacamos la expresión simbólica $d f=f^{\prime}(x)$. $d x$, la cual es utiliza, en cierta medida, por todas las demás $\mathrm{CO}$. 
En el tercer nivel de generalización y formalización ponemos a la $\mathrm{CO}_{3}$ : diferencial de Fréchet, el cual lo identificamos con la aplicación lineal; y en el cuarto nivel se encuentra la $\mathrm{CO}_{4}$ : diferencial en el análisis no estándar donde resaltamos a los "infinitesimales". Cabe aclarar que en la $\mathrm{CO}_{4}$ el concepto de aplicación lineal y aproximación de la $\mathrm{CO}_{3}$ son utilizados en el teorema del incremento del análisis no estándar. Los análisis obtenidos dan como resultado el modelo ontosemiótico de los significados del diferencial, reflejado en la Figura 6.

Figura 6: Modelo ontosemiótico de los significados del diferencial

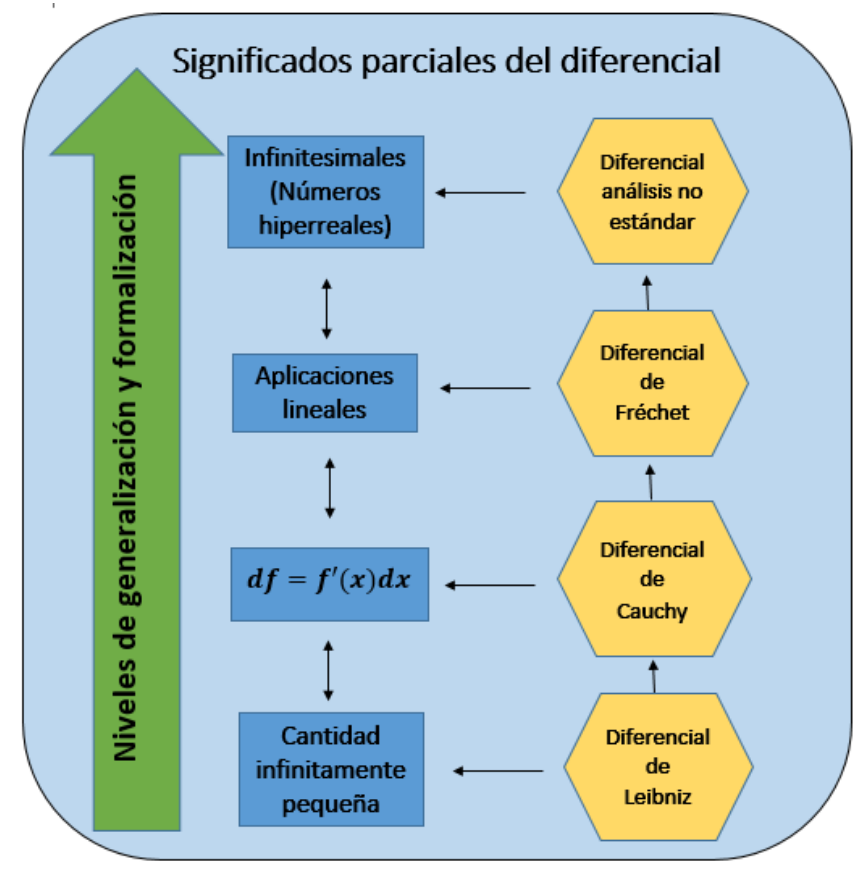

Fuente: Elaboración propia (2021)

Como se puede observar, $\mathrm{CO}_{1}, \mathrm{CO}_{2}$ y la $\mathrm{CO}_{3}$ están contemplados en la $\mathrm{CO}_{4}$ porque es posible pensarlos como casos particulares estableciendo ciertas condiciones en la utilización de los objetos matemáticos primarios. Esta característica está expresada por medio de las dobles implicaciones entre los objetos que destacamos en cada $\mathrm{CO}$. Cada una de las $\mathrm{CO}_{1}, \mathrm{CO}_{2}, \mathrm{CO}_{3}$ y la $\mathrm{CO}_{4}$ constituyen significados parciales del concepto diferencial.

\section{Reflexiones finales e implicaciones para la educación matemática}

El modelo ontosemiótico del diferencial presentado en esta investigación, basado en la perspectiva antropológica y pragmatista del EOS, constituye un aporte relevante para la didáctica del análisis matemático ya que es posible repensar la planificación curricular para mejorar las prácticas de enseñanza, diseñar secuencias didácticas argumentadas y afrontar las potenciales dificultades de aprendizaje. Así, el modelo, ofrece una visión sistémica pragmática del concepto, identificando las 
prácticas, lenguajes, definiciones, proposiciones, procedimientos y argumentos que engloban y caracterizan a un significado, sin reducirlo a una única posible representación del concepto (FONT, PINOFAN y BREDA, 2020). Además, brinda elementos claves que el profesor de matemática debería tener en cuenta para realizar una enseñanza idónea del concepto.

El reconocimiento de los diversos significados del concepto de diferencial junto con sus interconexiones constituye un aspecto importante de la faceta epistemica del conocimiento didácticomatemático del profesor de matemáticas ya que es requisito para realizar una enseñanza idónea de este concepto. Pero también, los análisis presentados se espera que sirvan de base al docente para realizar los análisis a priori requeridos para el diseño de tareas. En este sentido, la identificación de los objetos y procesos que intervienen y emergen en las prácticas matemáticas debería ser una competencia específica del profesor que le permitirá comprender la progresión de los aprendizajes, identificar potenciales conflictos semióticos, gestionar procesos de institucionalización y evaluar la competencia matemática de los estudiantes (GODINO et al., 2017).

\section{Reconocimiento.}

Trabajo realizado en el marco de los proyectos PGC2018-098603-B-I00 (MCIU/AEI/FEDER, UE), PFID-FID-2021-45 (Panamá), Grupo S60_20R - Investigación en Educación Matemática (Gobierno de Aragón y Fondo Social Europeo) y 16Q691-PI (FCEQyN - UNaM, Argentina). Los autores agradecen la colaboración en la lectura detallada al doctor Juan D. Godino.

\section{Referencias}

ARTIGUE, Michèle. Le passage de la différentielle totale à la notion d'application linéaire tangente. In: Procedures différentielles dans les enseignements de mathematiques et de physique au niveau du premier cycle universitaire (Annexe I). Université Paris 7: IREM et LDPES, 1989.

ARTIGUE, Michèle. La enseñanza de los principios del cálculo: problemas epistemológicos, cognitivos y didácticos. In: GOMEZ, Pedro (Ed.). Ingeniería Didáctica en Educación Matemática. Un esquema para la investigación y la innovación y el aprendizaje de las matemáticas. Una empresa docente y Grupo editorial Iberoamérica, p. 97-140, 1995.

ARTIGUE, Michèle; MENIGOUX, Jacqueline; VIENNOT, Laurence. Some aspects of students' conceptions and difficulties about differentials. European Journal of Physics, v. 11, n. 5, p. 262-267, 1990. DOI: 10.1088/0143-0807/11/5/002.

BOYER, Carl. B. The history of the calculus and its conceptual development. New York, USA: Dover, 1987. 
BOS, Hendrik Jan Maarten. Differentials, higher-order differentials and derivatives in the Leibnizian calculus. Archive for history of exact sciences, v. 14, n. 1, p. 1-90, 1974.

BURGOS, María; BUENO, Seydel; PÉREZ, Olga; GODINO, Juan D. Onto-semiotic complexity of the Definite Integral. Journal of Research in Mathematics Education, v. 10, n. 1, p. 4-40, 2021. DOI: 10.17583/redimat.2021.6778

BURGOS, María; GODINO, Juan D. Modelo ontosemiótico de referencia de la proporcionalidad. Implicaciones para la planificación curricular en primaria y secundaria. AIEM Avances de Investigación en Educación Matemática, n. 18, p. 1-20, 2020. DOI: 10.35763/aiem.v0i18.255.

CAUCHY, Augustin Louis. Cours d'analyse de l'Ecole Royale Polytechnique. París: de l'Imprimerie Royale, ETH Library Zurich, 1821. DOI: 10.3931/e-rara-26185.

CAUCHY, Augustin Louis. Résumé des leçons données à l'Ecole Royale Polytechnique sur le calcul infinitésimal. París: de l'Imprimerie Royale, ETH Library Zurich, 1823. DOI: 10.3931/e-rara-25962.

DRAY, Tevian; MANOGUE, Corinne A. Putting Differentials Back into Calculus. The College Mathematics Journal, v. 41, n. 2, p. 90-100, 2010. DOI: 10.4169/074683410X480195.

EDWARDS, Charles Henry. The historical development of the calculus. New York, USA: SpringerVerlag, 1979. DOI: 10.1007/978-1-4612-6230-5.

FONT, Vicenç; PINO-FAN, Luis R.; BREDA, Adriana. Una evolución de la mirada sobre la complejidad de los objetos matemáticos. Revista Paradigma, v. 41, p. 107-129, 2020. DOI: 10.37618/PARADIGMA.1011-2251.2020.p107-129.id846.

FONT, Vicenç; GODINO, Juan D.; GALLARDO, Jesús. The emergence of objects from mathematical practices. Educational Studies in Mathematics, n. 82, p. 97-124, 2013. DOI: 10.1007/s10649-0129411-0.

GODINO, Juan D. Construyendo un sistema modular e inclusivo de herramientas teóricas para la educación matemática. In: CONTRERAS, José Miguel; ARTEAGA, Pedro; CAÑADAS, Gustavo R.; GEA, María Magdalena; GIACOMONE, Belén; LÓPEZ-MARTÍN, María del Mar (Eds.), Actas del Segundo Congreso International Virtual sobre el Enfoque Ontosemiótico del Conocimiento y la Instrucción Matemáticos, 2017. Disponible en http://enfoqueontosemiotico.ugr.es/civeos.html

GODINO, Juan D.; BATANERO, Carmen. Significado institucional y personal de los objetos matemáticos. Recherches en Didactique des Mathématiques, v. 14, n. 3, p. 325-355, 1994.

GODINO, Juan D.; BATANERO, Carmen; FONT, Vicenç. The onto-semiotic approach to research in mathematics education. ZDM Mathematics Education, n. 39, p. 127-135, 2007. DOI: 10.1007/s11858006-0004-1.

GODINO, Juan D.; BATANERO, Carmen; FONT, Vicenç. El enfoque ontosemiótico: implicaciones sobre el carácter prescriptivo de la didáctica. Revista Chilena de Educación Matemática, v. 12, n. 2, p. 3-15, 2020. DOI: 10.46219/rechiem.v12i2.25.

GODINO, Juan D. et al. Why is the learning of elementary arithmetic concepts difficult? Semiotic tools for understanding the nature of mathematical objects. Educational Studies in Mathematics, v. 77, n. 2, p. 247-265, 2011. DOI: 10.1007/s10649-010-9278-X. 
GODINO, Juan D. et al. Enfoque Ontosemiótico de los Conocimientos y Competencias del Profesor de Matemáticas. Bolema: Boletim de Educação Matemática, v. 31, n. 57, p. 90-113, 2017. DOI: 10.1590/1980-4415v31n57a05.

GOMEZ, Alfonso. A look to differential. Journal of Physics: Conference Series. V International Conference Days of Applied Mathematics, v. 1414, n. 1, p. 1-7, 2019. DOI: 10.1088/1742$6596 / 1414 / 1 / 012001$.

GORDILLO, W.; PINO-FAN, L. R. Una Propuesta de Reconstrucción del Significado Holístico de la Antiderivada. Bolema: Boletim de Educação Matemática, v. 30, n. 55, p. 535-558, 2016. DOI: 10.1590/1980-4415v30n55a12.

HU, Dehui; REBELLO, N. Sanjay. Understanding student use of differentials in physics integration problems. Physical Review Special Topics - Physics Education Research, v. 9, n. 2, p. 1-14, 2013. DOI: 10.1103/PhysRevSTPER.9.020108.

KEISLER, H. Jerome. Elementary calculus. An infinitesimal approach. California, USA: University of Wisconsin, 2000.

KLEINER, Israel. History of the infinitely small and the infinitely large in calculus, with remarks for the teacher. In Excursions in the History of Mathematics. Birkhäuser Boston, p. 67-101, 2012. DOI: 10.1007/978-0-8176-8268-2_4.

KLEINER, Israel. History of the Infinitely Small and the Infinitely Large in Calculus. Educational Studies in Mathematics, n. 48, p. 137-174, 2001. DOI: 10.1023/A1016090528065.

LOBO, Rogério Santos. Utilizando a Derivada para inferir sobre a velocidade de contaminação do novo Coronavírus: uma possibilidade para as aulas de Cálculo. Revemop, v. 2, e202021, p. 1-18, 2020. DOI: 10.33532/revemop.e202021

LÓPEZ-GAY, Rafael Lucio-Villegas. La introducción y utilización del concepto de diferencial en la enseñanza de la física. Análisis de la situación actual y propuesta para su mejora. 2001. (Tesis doctoral). Universidad Autónoma de Madrid, España.

LÓPEZ-GAY, Rafael; MARTÍNEZ SÁEZ, Julio; MARTÍNEZ TORREGROSA, Joaquín. Obstacles to mathematization in physics: The case of the differential. Science \& Education, n. 24, p. 591-613, 2015. DOI: 10.1007/s11191-015-9757-7.

MARTÍNEZ-TORREGROSA, Joaquín; LÓPEZ-GAY, Rafael; GRAS-MARTÍ, A. Mathematics in physics education: scanning historical evolution of the differential to find a more appropriate model for teaching differential calculus in physics. Science \& Education, v. 15, n. 5, p. 447-462, 2006. DOI: 10.1007/s11191005-0258-y.

MARTÍNEZ-TORREGROSA, Joaquín; LÓPEZ-GAY, Rafael; GRAS-MARTÍ, Albert; TORREGROSAGIRONÉS, Germán. La diferencial no es un incremento infinitesimal. Evolución del concepto de diferencial y su clarificación en la enseñanza de la física. Enseñanza de las Ciencias, v. 20, n. 2, p. $271-$ 283, 2002.

OLDENBURG, Reinhard. Differentiale als Prognosen. Journal für Mathematik-Didaktik, n. 37, p. 5582, 2016. DOI: 10.1007/s13138-016-0096-2. 
ORTON, Anthony. Students' understanding of differentiation. Educational Studies in Mathematics, n. 14, p. 235-250, 1983. DOI: 10.1007/BF00410540.

PINO-FAN, Luis R.; GODINO, Juan D.; FONT, Vicenç. Faceta epistémica del conocimiento didácticomatemático sobre la derivada. Educação Matemática Pesquisa, v. 13, n. 1, p. 141-178, 2013.

PULIDO, Ricardo. Un estudio teórico de la articulación del sable matemático en el discurso escolar: la transposición didáctica del diferencial en la física y la matemática escolar. 1997. (Tesis doctoral). Cinvestav, México.

PULIDO, Ricardo. La enseñanza de los diferenciales en las escuelas de ingeniería desde un enfoque socioepistemológico. Relime: Revista Latinoamericana de Investigación en Matemática Educativa, v. 13, n. 4, p. 85-97, 2010.

ROBINSON, Abraham. Non-standard analysis. Los Ángeles, USA: North-Holland Publishing Company Amsterdam, 1966.

TALL, David. Comments on the difficulty and validity of various approaches to the calculus. For the Learning of mathematics, v. 2, n. 2, p. 16-21, 1981a.

TALL, David. Intuitions of infinity. Mathematics in School, v. 10, n. 3, p. 30-33, 1981b.

TAYLOR, A. The differential: nineteenth and twentieth century developments. Archive for History of Exact Sciences, v. 12, n. 4, p. 355-383, 1974.

VALDIVÉ, Carmen; GARBIN, Sabrina. Estudio de los esquemas conceptuales epistemológicos asociados a la evolución histórica de la noción de infinitesimal. Relime: Revista Latinoamericana de Investigación en Matemática Educativa, v. 11, n. 3, p. 413-450, 2008.

VERÓN, Manuel Alejandro. Análisis ontosemiótico de los significados del concepto de diferencial. 2020. (Tesis de maestría). Universidad de Granada, España. DOI: 10.13140/RG.2.2.17929.16489.

WILHELMI, Miguel R.; GODINO, Juan D.; LACASTA, Eduardo. Configuraciones epistémicas asociadas a la noción de igualdad de números reales. Recherches en Didactique des Mathematiques, v. 27, n. 1, p. 77-120, 2007. 